\title{
La producción de textos escritos en estudiantes de dos universidades públicas con el modelo intercultural
}

\section{Production of texts in students from two public universities with intercultural model}

\author{
José Bastiani-Gómez ${ }^{1}$ \\ Universidad Intercultural de Chiapas \\ División de Procesos Sociales \\ San Cristóbal de Las Casas, Chiapas, México \\ jbastiani@unich.edu.mx \\ María Minerva López-García ${ }^{2}$ \\ Universidad Autónoma de Chiapas \\ Facultad de Humanidades, Campus VI \\ Tuxtla Gutiérrez, Chiapas, México \\ minerva@unach.mx
}

Recibido: 10 marzo 2015 Aceptado: 22 octubre 2015 Corregido: 11 noviembre 2015

Resumen: El objetivo de este trabajo consiste en identificar y analizar las características de la comunicación escrita del estudiantado de la Licenciatura en Lengua y Cultura de la Universidad Intercultural de Chiapas y de la Licencicatura en Educación Preescolar y Primaria de la Universidad Pedagógica Nacional, Unidad 071, de Tuxtla Gutiérrez, Chiapas y su incidencia en la formación intercultural. El estudio es de carácter exploratorio y descriptivo. Hace uso de la técnica de la encuesta, la observación participante y la entrevista grupal a una muestra por conveniencia de 83 estudiantes. Los datos obtenidos permitieron reflexionar y discutir sobre las características de la producción de textos escritos a partir del análisis de sus productos y experiencias de aprendizaje lectoescritoras. Los resultados indican que el estudiantado presenta una comunicación escrita con deficiencias en la enunciación de los temas y la congruencia en la redacción de textos, entre otros problemas. Reconocen que han experimentado una formación escolar deficiente y ajena a la procedencia lingüistica de origen étnico. Las dificultades identificadas obedecen, entre otras causas, a razones de orden didáctico ausentes en la práctica docente, a precarias condiciones de organización escolar, a la escasa reflexividad sobre la enseñanza bilingüe, al poco compromiso ético de quien asesora y a una deficiente formación del profesorado y equipo de asesoría académica, en ambos programas. Se sugiere impulsar una formación docente intercultural que promueva estrategias didácticas tanto en la lengua indígena como en español en los programas de estudio para alcanzar resultados favorables de calidad en su formación.

Palabras claves: Comunicación escrita, bilingüismo, educación superior, experiencias lectoescritoras, educación intercultural.

1 Doctor en Ciencias en Ecología y Desarrollo Sustentable en El Colegio de la Frontera Sur. Maestro en Ciencias en Educación Indígena por la Universidad Autónoma de Chiapas y Licenciado en Sociología por la Universidad Juárez Autónoma de Tabasco. Profesor investigador en la Universidad Intercultural de Chiapas, miembro del SNI, nivel 1. Temas de investigación: educación, interculturalidad, educación ambiental y comprensión lectora. E-mail: bastianijose14@hotmail.com, Publicaciones recientes: Bastiani Gómez, José et al. (2012), "Política educativa indígena: Práctica docente, castellanización, burocracia y centralización de la educación como limitaciones del éxito pedagógico en la región Ch’ol, Chiapas”, en Perfiles Educativos, 34(135); Bermúdez Urbina, Flor Marina et al. (2012), "Concepciones sobre desarrollo sustentable en mujeres indígenas de la Universidad de Ciencias y Artes de Chiapas, Sede Motozintla”, en Redhes, año III, núm. 6, julio-diciembre 2011. Disponible en: http://www.derecho.uaslp.mx/Documents/Revista\%20REDHES/N\%C3\%BAmero\%206/Redhes6-03.pdf; Bastiani Gómez, José y Reyna Moguel Viveros (2011), "Exclusión e inclusión del modelo de educación superior intercultural: En el caso de la Unich", en Signos Lingüísticos, 12 y 13, julio-diciembre, 2010/enero-junio, 2011.

2 Docente de la Facultad de Humanidades, Campus VI de la Universidad Autónoma de Chiapas. Licenciada en Psicología, Universidad Veracruzana. Maestra en Educación Superior, Universidad Autónoma de Chiapas y Doctora en Educación, Universidad del Sur. Miembro del Sistema Nacional de Investigadores, Nivel I. Temas de investigación: educación, interculturalidad, comprensión lectora, estrategias de aprendizaje, educación especial. 


\begin{abstract}
The objective of this study was to identify and analyze the characteristics of the written communication of the Language and Culture students at Chiapas Intercultural University and the Early Education and Elementary School Education students at the National Pedagogical University, Unit 071, in Tuxtla Gutierrez, Chiapas and its relation with their intercultural training. The nature of the study is exploratory and descriptive and used a survey, participant observation and a group interview with a convenience sample of eighty-three students which allowed the reflection on and the discussion about the characteristics of the students' written production based on the analysis of their written production and their reading-writing experiences. Results indicate that the most serious problems are topic development and coherence. Students claim that they have received poor schooling which has ignored their ethnical background. These difficulties are due to lacks in their teaching training, the precarious condition of school organization, the lack of reflection on bilingual education, the advisor's lack of ethical commitment and the deficient training received by their professors and advisors. The article argues that teacher education must be intercultural, including teaching strategies appropriate for both the indigenous language and Spanish.
\end{abstract}

Keywords: written communication, bilingualism, higher education, lectoescritoras experiences, intercultural education.

El modelo de formación intercultural en México tiene como uno de sus principales propósitos formar profesionales con un sólido dominio de la escritura, tanto en español como en una lengua materna, para promover el bilingüismo; no obstante, el cumplimiento de tal exigencia se torna complejo en la enseñanza.

En el caso de la educación primaria, a través del documento Parámetros curriculares de la Asingatura de Lengua Indígena, se hace énfasis en la enseñanza de la lengua indígena en la que el estudiantado pueda construir estas competencias bilingües (SEP, 2008). Igualmente se encuentra la recomendación sobre este aspecto en los contenidos curriculares de las carreras de la Universidad Intercultural de Chiapas (UNICH), en la que se espera que a través de la impartición de cursos de lengua indígena su alumnado pueda, al término de su formación, ser bilingüe ${ }^{3}$.

Para el caso de las Normales y la Universidad Pedagógica Nacional (en adelante UPN), los programas de estudio hacen énfasis en un un perfil de egreso, en el dominio de competencias bilingües (Secretaría de Educación Pública [SEP], 2004; Universidad Intercultural de Chiapas [UNICH], 2012b; Universidad Pedagógica Nacional [UPN], 1990). Los planes de estudio mencionados consideran que sin el desarrollo de un bilingüismo funcional será difícil que las comunidades de la población estudiantil puedan ser parte de la construcción y convivencia de una sociedad del conocimiento que utilice la lengua escrita y oral como elemento para el diálogo intercultural.

3 En el año 2005, en la UNICH se crearon los programas de estudio para la enseñanza de las lenguas indígenas como prioridad en la formación de estudiantes sin que se haya llegado de manera formal a la elaboración de un programa curricular en cuyo eje transversal estuviera la lengua indígena. Sin embargo, para el año 2010, la Secretaría Académica de la Universidad impulsó la reformulación_de los Planes y Programas de Estudio por competencias en todas las carreras. Esta nueva concepción curricular, que a lo largo de tres años aproximadamente de trabajo institucional, ha conllevado a la redefinición del desarrollo de la adquisición de las competencias de lectoescritura de las lenguas originarias, estableciendo la enseñanza de estas de forma curricular: tsotsil, tseltal, ch'ol y zoque en todas las carreras. En la de Lengua y Cultura, en donde el alumnado tiene como eje curricular formativo el de la lengua indígena, aparecen dos líneas de especialización que se cursan en los tres últimos semestres (sexto, séptimo y octavo) que se llama Traducción. Aunque esta especialización es un avance en el logro formativo de alumnos y alumnas, además de los cursos de lengua indígena que se imparten, aún no se cuenta con un programa formal metodológico que contribuya al desarrollo y establecimiento de manera transversal de lengua en el currículum de esta carrera. Véase el mapa curricular versión por competencias del año 2013 que hace esta aclaración en las líneas formativas de la carrera Lengua y Cultura. 
En el año 2005, con la apertura de la Licenciatura en Lengua y Cultura de la Universidad Intercultural de Chiapas, se abrió un espacio para la formación profesional de jóvenes indígenas y población joven mestiza; en este proceso de formación el plan de estudios consideraba como prioridad que las lenguas indígenas pudieran obtener un estatus social igualitario en los diversos ámbitos de comunicación y, con ello, promover una diálogo amplio entre los diferentes actores a partir de las diferencias, por su origen cultural. Es importante señalar que aunque las actividades de enseñanza se realizan en español, adicionalmente el estudiantado cursa asignaturas relacionadas con la adquisición y perfeccionamiento de una lengua indígena, puesto que parte de estos grupos solo habla el español, como se verá en los datos más adelante. Regularmente, se oferta por semestre la enseñanza, por lo menos, de dos lenguas indígenas para que quienes no las conocen puedan acceder a su aprendizaje. Por otra parte, se espera que el estudiantado pueda ser capaz de comunicarse oralmente y por escrito en español de forma adecuada, así como comprender de forma oral y escrita para formarse sólidamente en la licenciatura que han elegido cursar.

Aunque no es el objetivo de este texto realizar una valoración sobre el éxito que estudiantes de Lengua y Cultura han tenido al egresar, sí pensamos que existe una estrecha relación entre el desarrollo de la comunicación escrita antes mencionada y sus posibilidades de empleo al concluir los estudios universitarios. A partir de la observación y el contacto con el estudiantado egresado hemos podido evidenciar que a ocho años de haber iniciado la carrera, el índice de eficiencia terminal y titulación es bajo ${ }^{4}$, ya que un número importante de personas egresadas no ha podido titularse ni emplearse. Lo primero, que nos atañe directamente, puede deberse al pobre desarrollo de la comunicación escrita, así como a la formación metodológica y teórica para llevar a cabo una investigación que se traduzca en un informe para la titulación a través de esta vía.

El estudiantado logra plasmar sus ideas con muchas dificultades en un trabajo académico (esta situación no es exclusiva de esta población sino que es generalizada, pero esa discusión cabrá en otro momento). Estas dificultades han motivado nuestro interés por indagar sobre las características de la comunicación escrita del estudiantado formado desde el enfoque intercultural: conocer sus fortalezas y limitaciones en esta práctica es necesario para tomar decisiones futuras en el ámbito de la competencia docente y curricular que nos atañe. A manera de supuesto que guió nuestra investigación, planteamos que la enseñanza para la formación bilingüe e intercultural en la cual es formada la población estudiantil de la Licenciaura en Lengua y Cultura así como los de Educación Preescolar y Primaria Indígena, no ha sido suficiente para afrontar las exigencias académicas en el nivel superior y, como un fenómeno en cascada, las repercusiones se reflejan en un pobre rendimiento académico.

Los perfiles de egreso, como consecuencia, no se logran como se espera, puesto que las debilidades encontradas en la formación imposibilita que el estudiantado egresado pueda estar suficientemente capacitado para desarrollar estrategias en la enseñanza de lenguas

4 El informe estadístico de la oficina de Área de Control Escolar y el Departamento de Planeación de la UNICH señalan que han egresado 976 alumnos y alumnas de cuatro generaciones de estudiantes en cuatro carreras: Lengua y Cultura, Comunicación Intercultural, Desarrollo Sustentable y Turismo Alternativo (2005-2009; 2006-2010; 2007-2011 y 2008-2012). El porcentaje de eficiencia terminal de cuatro cohortes generacionales fue de $67.82 \%, 80.98 \%$, $62.70 \%$, y $63.66 \%$, respectivamente. Sin embargo, el índice de titulación que se tiene de cuatro carreras en cuatro cohortes a la fecha de consulta de los datos es de 21.2\%. Ambos datos siguen siendo bajos. Para mayor información, véase UNICH (2012). Informe de rectoría enero-diciembre 2012, Universidad Intercultural de Chiapas, en http://www.unich.edu.mx/informe-rector/, fecha de consulta 5 de abril de 2013.- 
originarias $^{5}$, como es el caso de personal docente futuro de educación preescolar y primaria indígena, porque no han vivido la experiencia en su formación. Esto significa que tienen poco que ofrecer a sus estudiantes en un futuro cercano.

En América Latina la política educativa dirigida a los pueblos originarios, que ha tenido como principal fundamento la castellanización, se ha visto influida por presiones de organismos que le exigen elevar su eficiencia, expresada en los pobres resultados de indicadores educativos, los enfoques de educación indígena intercultural para el nivel de educación superior aún no logran crear las metodologías y las estrategias de enseñanza del español (López, 2003) en contextos educativos en donde solo se privilegia el español.

Las debilidades en la adquisición de la lectura y la escritura tanto en español como en alguna lengua indígena se presentan desde los niveles básicos, continúan durante el bachillerato y no se resuelven al llegar a la educación superior, como en el caso de estudiantes de la LEPEPMI' 90, que sigue siendo una de las principales problemáticas no atendidas con la propiedad que se merece, aunque se sugiera en el perfil de egreso, que saldrán con una sólida competencia lingüística de forma bilingüe.

La formación docente que recibe el profesorado en servicio, con el programa curricular de la LEPEPMI'90, dentro del eje disciplinar antropológico-lingüístico (UPN, 1990), aún no se ha constituido en una posibilidad formal de contribuir en el desarrollo del plurilingüismo en México (Pérez et al., 2012). Las aportaciones de Pérez et al. (2012), respecto a los procesos de formación de profesionales en el campo de la enseñanza de las lenguas y, concretamente, de la lenguas indígenas en el aula para potenciar su uso como medio pedagógico y fortalecer el desarrollo del bilingüismo equilibrado, no se han recuperado en el trabajo cotidiano de las aulas, dando lugar a una pobre didáctica de las lenguas extranjeras y nacionales en la educación superior $^{6}$. La escolaridad del estudiantado aparece matizada por trayectorias escolares individuales en donde el sistema educativo mexicano ha privilegiado la castellanización, y sus efectos en el campo superior son que la comunicación oral y escrita, tanto en lengua indígena como en castellano, sigue siendo un punto inconcluso para fortalecer las relaciones de comunicación social entre estudiantes.

El trabajo de Hamel, Brumm, Carrillo, Loncon, Nieto y Castellón (2004) en la región purépecha de Michoacán constata la complicada tarea que ha propiciado la castellanización en educación primaria, para dar paso a un bilingüismo sustractivo caracterizado por el

5 Los grupos de estudiantes de LEPEPMI'90 en sexto, séptimo y octavo semestre a través de los cuatro campos formativos (social, lengua, matemáticas y naturales) enfatizan, a lo largo de los cursos, que en tres semestres concretarán la elaboración del documento recepcional de Propuesta Pedagógica como proceso formativo y de titulación. El claustro docente, bajo los lineamientos pedagógicos y acuerdos tomados, privilegia como única opción de titulación la Propuesta Pedagógica. Ha habido casos excepcionales de titulación con otras modalidades como de tesina o tesis, pero particularmente el estudiantado debe realizar una Propuesta Pedagógica de Titulación en alguno de los campos formativos curriculares que se ha mencionado. En el campo de la lengua, los trabajos recepcionales de Propuesta Pedagógica que presentan los alumnos y las alumnas consideran el fortalecimiento de la lectoescritura de la lengua indígena y del español, intentando poner en las estrategias didácticas el uso y manejo de la comunicación oral, escrita, comprensión oral y comprensión lectora en ambas lenguas (español y lengua indígena). Se logra observar, a lo largo de las trayectorias escolares, las dificultades que tiene el alumnado por escribir y plasmar su ideas, lo que impide que cumplan con las expectativas de titulación en tiempo y forma de la Lepepmi’90 en la Universidad Pedagógica Nacional, Unidad 071, de Tuxtla Gutiérrez, Chiapas, México.

6 Las autoras señalan que se están haciendo esfuerzos importantes en la elaboración de programas curriculares para la enseñanza de las lenguas nacionales y extranjeras, pero que en el ámbito de la didáctica de las leguas para ambos contextos todavía no se observan avances importantes, y aún es más grave el caso de las lenguas indígenas, que no logran ubicarse en el terreno de la enseñanza con buenas metodologías y una sólida formación linguopedagógica. Hay que admitir que LEPEPMI90 no está dirigido a formar profesionistas en el campo de la enseñanza de la lengua indígena, ya que es un planteamiento curricular disciplinario que en su afán de transdisciplinariedad tiene la intención de que los alumnos y alumnas puedan contribuir en la elaboración de propuestas pedagógicas en el campo de la lengua indígena y el castellano. 
desplazamiento vertiginoso de las lenguas originarias. En la UPN sucede esta misma situación, con una arista comunicativa diferente por el nivel y modalidad de estudio: en los procesos de formación docente indígena se ha ido configurando una práctica de castellanización que se traduce en una práctica bilingüe que refuerza aquella vieja concepción bicultural y bilingüe de la década de los ochenta, fuertemente impulsada por la Dirección General de Educación Indígena (DGEI) en México.

Aunque haya un afán en el currículo de LEPEPMI'90 de impulsar una formación docente intercultural bilingüe, su desarrollo aún sigue siendo cuestionado por la incapacidad de propiciar una formación pertinente de los pueblos originarios y para estos. En el alumnado, la falta de dominio en la lectura-escritura de la lengua castellana se refleja en la ausencia de reflexiones cognitivas sobre los textos, convirtiéndose en una fase inconclusa del desarrollo del modelo pedagógico intercultural en la universidad. La reiterada omisión del manejo de signos de puntuación y ortográficos, así como la escasa estructuración de las oraciones en su componente morfológico y sintáctico, posiblemente impida que el estudiantado se convierta en un sector lector que pueda adquirir y manejar el lenguaje pertinente que le permita comunicarse en un mundo de relaciones económicas y culturales como el que depara la globalización.

Abajo y Carrasco (2011), recuperando la teoría ecológica cultural Ogbu, señalan que los conflictos escolares de reprobación y eficiencia terminal, entre otros, que experimentan las minorías étnicas, se deben a causas estructurales del sistema social que impide un proceso de escolarización de éxito en estas minorías. En ese sentido, la población estudiantil de origen indígena está constituida por sujetos, históricamente hablando, que han logrado conformar una identidad bajo un conjunto de relaciones de desigualdad estructural que se manifiesta de diversas formas. Por eso, esta historicidad escolarizada marcada por procesos de exclusión, minorización, pobreza y marginación propicia un sinnúmero de prácticas alfabetizadoras que ponen al descubierto debilidades educativas entre el alumnado indígena de ambas universidades que intentan poner en práctica el modelo intercultural. El modelo intercultural tiene como objetivo que los alumnos y alumnas indígenas puedan escribir y leer en la lengua materna y en castellano, creando relaciones educativas simétricas en los procesos escolares (SEP-CGEIB, 2006, p. 36).

La formación escolar atravesada por el alumnado indígena universitario se caracteriza por la ausencia de alfabetización en las lenguas indígenas de su filiación étnica y social. La carencia de un método de forma sistemática que promueva la adquisición de la lectoescritura en lengua indígena ha contribuido a la pérdida y extinción de la lengua originaria. En el ámbito microescolar, las implicaciones de las desigualdades estructurales en la apropiación de la lengua se reflejan en el manejo inadecuado de conocimientos gramaticales, fonológicos, sintácticos, morfológicos y semánticos. Ello ha propiciado que el estudiantado no logre reflexionar sobre el papel de la lengua en los procesos de transmisión sociocultural. La correcta utilización de los adjetivos, pronombres, plurales y singulares, el manejo de la glotal, de las consonantes y vocales, así como de los constituyentes de la oración (verbo, objeto y sujeto) en los procesos de escrituralidad, ocasiona que difícilmente puedan lograr el dominio de la competencia comunicativa escrita en la lengua originaria.

Por otra parte, los procesos de alfabetización en lengua indígena se han anclado en las trayectorias escolares del estudiantado como expresiones orales. Esto ha desembocado en que los conocimientos se socialicen desde la educación básica hasta la educación media superior, 
en una tendencia de transmisión oral. Atendiendo a los señalamientos de Ogbu (Abajo y Carrasco, 2011), esta situación de desventaja que experimenta la población estudiantil por su origen étnico se caracteriza por condiciones de pobreza social y educativa. Podríamos señalar que la labor escolar realizada por estudiantes de las dos universidades públicas que participaron en este trabajo de investigación, a pesar de que hacen todo lo posible por insertarse en la estructura educativa y participar con limitaciones de capital educativo, está en desventaja frente al alumnado que solo habla el castellano. Las trayectorias escolares de la mayoría de la población estudiantil de la UNICH y la UPN están marcadas por sus historias de vida cultural que se encuentran entrelazadas por las relaciones comunicativas asimétricas, situación que los limita para poder participar en un mundo globalizado.

\section{Los programas de estudio de la Universidad Intercultural de Chiapas y la Universidad Pedagógica Nacional, Unidad 071 y la elaboración de textos descriptivos}

En el año 2005, en un impulso a la educación indígena bilingüe intercultural, se crea la Universidad Intercultural de Chiapas, con el objetivo de que la población indígena pueda acceder a la educación superior desde el supuesto de que se valore y se enseñen las lenguas indígenas y el español, para poder construir relaciones educativas interculturales. Muestra de esto es la proporción de estudiantes atendido durante el ciclo escolar enero-junio 2012, a una población de 1,450, de la cual 480 eran tseltales, 195 tsotsiles, 605 mestizos y mestizas, 75 ch'oles, 78 tojolobales, 22 zoques, 12 k'anjobales y un mixteco, provenientes de diferentes comunidades indígenas de Chiapas, lo que da cuenta de la interacción que se da entre el estudiantado a partir de su lengua de origen, la que, al mismo tiempo, lo es de su cultura originaria.

La preocupación por valorar las lenguas indígenas se observa en el caso del Plan y Programas de estudio de la carrera de Lengua y Cultura, con una duración de cuatro años en donde se espera, de acuerdo con el perfil de egreso, que sus estudiantes, al finalizar su formación, tendrán un sólido manejo de competencias comunicativas orales y escritas en las dos lenguas: el español y la lengua indígena (UNICH, 2007; UNICH, 2012a).

Por su parte, desde hace más de tres décadas, la UPN es considerada como una de las instituciones de educación superior más importante en la formación de docentes en el campo de la educación básica. En la década de los noventa del siglo pasado, la Universidad Pedagógica Nacional, Unidad Ajusco, diseñó un programa de estudios para atender la diversidad étnica de sus docentes. Para lograr este propósito puso en marcha la Licenciatura en Educación Preescolar y Primaria Indígena (LEPEPMI'90) con el fin de formar maestras y maestros indígenas en las regiones multiculturales de México, caracterizadas por la presencia de diversos grupos indígenas. En Chiapas, la dirección de la UPN, Unidad 071, retomó el Plan y Programas de Estudio de LEPEPMI'90, con lo que inició una nueva etapa en la formación de docentes indígenas. ${ }^{7}$

El estudiantado que cursa estudios de Licenciatura en Educación Preescolar y Primaria

7 Antes del año 1990 en la Universidad Pedagógica Nacional, Unidad 071, de Tuxtla Gutiérrez, Chiapas, el profesorado indígena que laboraba para la Dirección de Educación Indígena se profesionalizaba en algunos programas docentes de educación primaria como la Licenciatura en Educación Primaria, Plan 1980 de la UPN en donde asistía un profesorado de las diversas regiones étnicas de Chiapas. También las escuelas normales en la entidad chiapaneca ofrecían la formación de docentes de primaria, pero, no era licenciatura la formación sino el título que expedía decía Profesor de Primaria y para ingresar a profesionalizarse en la Normal lo hacían con estudios de secundaria. La reforma educativa de 1992 en México elevó a rango los estudios de profesorado de primaria a licenciatura como opción política para mejorar la habilitación pedagógica del magisterio nacional y, en donde, de igual manera se formarían los profesores y profesoras de educación indígena. 
Indígena en el Plan 1990 se forma durante cuatro años consecutivos, al término de los cuales se espera que el estudiantado egresado obtenga una sólida formación en el área de la lengua materna y el español, que le permita innovar su práctica docente; situación que, de entrada, se torna compleja por la diversidad de estudiantes que dominan lenguas maternas distintas. En todos los casos, debería priorizarse alguna en particular para su enseñanza.

Los ejes curriculares del Plan de Estudios mantienen una relación articulada con las áreas de enseñanza en la educación básica del Plan y Programas de Estudio 1993 (SEP, 1993), es decir, la formación que obtienen en la LEPEPMI'90 les otorga una formación en el conocimiento de los enfoques psicopedagógicos y en el dominio de las lenguas indígenas y español, con el propósito de que su desempeño en las escuelas bilingües de educación preescolar y primaria indígena se desarrolle con el enfoque comunicativo de la lengua (SEP, 2011).

\section{Marco teórico: Punto de articulación de la teoría para abordar el problema de investigación}

El lenguaje, tanto oral como escrito, se produce entre un ente emisor y un ente receptor que dialogan en un contexto social y cultural determinado, es decir, la apropiación de la lengua tiene un carácter social. En este proceso intervienen, además, diversos factores de naturaleza cultural y neurológica que determinan el desarrollo de los procesos cognitivos de las personas en el dominio y aprendizaje de diversas competencias comunicativas, lingüísticas y pragmáticas (Ruiz, 2000).

Los avances de investigación llevados a cabo desde diversas concepciones (psicolingüística, sociolingüística o psicología cognitiva) aluden a diversos factores sociales y ambientales que inciden en la adquisición de segundas lenguas. Posiciones encontradas defienden ciertos mecanismos básicos, como el conocimiento de nociones gramaticales necesarias para construir un texto de manera oral y escrita, mientras que otras vertientes de análisis lingüístico señalan que los entornos naturales y sociales son también espacios que redefinen la construcción del habla oral y escrita para el logro de la competencia comunicativa, con la que entablar una comunicación fluida y eficaz (Areizaga, 2000; Ruíz, 2000).

En resumen, desde la década de los setenta y ochenta del siglo XX, las concepciones teóricas del input monitor, modelo de procesamiento de la atención, modelo multidimensional y modelo de aculturación, han aportado interesantes discusiones en las que no hay una única concepción de la forma en que las personas puedan aprender la segunda lengua (Ruiz, 2000). En consecuencia, todas estas teorías apuntalan que la adquisición de una segunda lengua implica diversos factores sociales, ecológicos, ambientales, pedagógicos, didácticos, neurológicos y culturales que contribuyen a que las personas aprendan la lengua escrita y oral, además de los componentes gramaticales para establecer la comunicación. La lingüística del texto ha hecho importantes precisiones para su elaboración, señalando que la expresión oral y escrita es mediada por diversos componentes de naturaleza social y psicológica que determinan la forma en que se construye el conocimiento del lenguaje. El estudio de la adquisición de las segundas lenguas ha implicado conocer cómo en las comunidades escolares aprehenden el lenguaje y cómo se desarrolla para asegurar el dominio de la competencia comunicativa (SEP, 2011). 
La escritura es entendida como la capacidad que tienen las personas de comprender y producir discursos, por lo que el texto y el discurso son claves para aproximarse a lo que sucede en la comunicación (Ruiz, 2000). En nuestro país, desde hace varias décadas, la Secretaría de Educación Pública (SEP) viene trabajando con la población indígena el enfoque de competencia comunicativa para el aprendizaje de la segunda lengua: el castellano. Esta política privilegia que se adquieran las competencias comunicativas de expresión oral, expresión escrita, comprensión oral y la reflexión de la lengua en el sentido de que el alumando pueda agenciarse de la estructura gramatical, fonológica, sintáctica y semántica como principios necesarios para la elaboración de diversos discursos textuales. En la estructuración del lenguaje escrito y oral se toman en cuenta aspectos anímicos y afectivos indispensables para el establecimiento del aprendizaje de la lengua y de sus códigos respectivos.

Es así como, a partir del reconocimiento de aspectos fonológicos, sintácticos y morfológicos, se evoca la capacidad metacognitiva de los estudiantes para estructurar una idea, una frase, una oración, el manejo de los verbos, del sujeto y del predicado, y se hace comprensible la elaboración de los textos (Gómez, 1997). Las reformas, desde la educación básica hasta la superior, están poniendo un fuerte énfasis en que los grupos estudiantiles puedan egresar de la formación profesional con una sólida competencia comunicativa que les permita adquirir diversos procedimientos metodológicos que fortalezcan sus capacidades en el área de lengua. La eficiencia de los sistemas escolares depende más del desarrollo de las habilidades de compresión lectora y de la escritura de la población estudiantil, principios básicos indispensables para construir una ciudadanía política y cultural.

La política educativa impulsada por el gobierno federal mexicano, a través del Plan Nacional de Desarrollo 2007-2012 y el Programa Sectorial de Educación 2007-2012, la Alianza por la Calidad de la Educación de 2008 y la Reforma de la Educación Básica (RIEB), estableció elevar la calidad educativa, a partir de la opción del modelo educativo por competencias. En este se establece la formación integral de alumnos y alumnas para poder insertarse en la vida económica del mundo contemporáneo (SEP, 2010). Es así como las competencias educativas sitúan a las generaciones de estudiantes mexicanos en la adquisición:

...de conocimientos, habilidades, actitudes y valores para el logro de propósitos en contextos y situaciones diversas... por lo que la educación básica debe lograr y contribuir a la formación de ciudadanos con estas características mediante el desarrollo de competencias como propósito educativo central. (SEP, 2010, p. 5)

Esta disposición curricular que propone como prioridad el desarrollo de un conjunto de conocimientos, destrezas y habilidades cognitivas, tanto para el campo de la educación básica como media superior y superior, enfatiza un saber hacer que son las habilidades para poder acceder al conocimiento. Además de que en este proceso escolar se asimilan y se desprenden las consecuencias de ese hacer educativo a través de las actitudes y valores necesarios para que el estudiantado aprenda a vivir en una sociedad moderna y compleja (Fernández, 2005; Frade, 2009; SEP, 2010). En ese sentido, el dominio de competencias en el ámbito de la comunicación escrita y oral de las lenguas originarias y del español resulta de valor pedagógico y cultural para afrontar las necesidades de desarrollo en la sociedad del conocimiento (SEP, 2011). El 
dominio de habilidades sociales de comunicación bilingüe entre estudiantes indígenas de la Universidad Pedagógica Nacional y la Universidad Intercultural de Chiapas se ubica como una de las principales prioridades en su formación.

\section{Procedimientos metodológicos}

Esta investigación optó por la perspectiva metodológica cualitativa, que consiste en descubrir y analizar (Aubry, 2011; Goetz y LeCompte, 1988; Woods, 1998) el nivel de comunicación escrita en la elaboración de textos en español que poseen estudiantes en dos instituciones de Educación Superior en Chiapas: la Universidad Intercultural de Chiapas y la Universidad Pedagógica Nacional 071, cuyos programas educativos poseen el enfoque intercultural. La interpretación se convierte en un acto que deviene en el análisis de los datos de inscripciones que realiza el estudiantado, más las categorías construidas a partir de la información obtenida de los trabajos escritos y las descripciones realizadas de los grupos de alumnos y alumnas, considerados como espacios de análisis e indagación que permitieron, junto a los constructos teóricos, asumir una posición objetiva e interpretativa (Bertely, 2002).

En ese sentido, la información proveniente de disciplinas científicas que se inscriben en el campo de la lingüística: psicolingüística, sociolingüística, semiótica y pedagogía, han profundizado en el análisis y comprensión de diversos procesos tanto cognitivos como socioculturales que intervienen en la apropiación del lenguaje oral y escrito. La información obtenida de las consultas a fuentes bibliográficas fue contenida y procesada en fichas de trabajo con el fin de ordenar y sistematizar la información que da cuenta de las implicaciones teóricas y empíricas en la producción de textos escritos.

Durante un periodo de tres meses se dio seguimiento a la producción de textos escritos de 63 estudiantes que tienen como lengua materna al tsotsil, tseltal, chol, además del zoque y de 20 que solo hablan español. Este seguimiento permitió valorar las características que presentan los textos que redactaron, así como sus debilidades y fortalezas en cuanto a la apropiación de la lectoescritura en español.

Para obtener la información empírica se elaboró un cuestionario que consta de dos partes: una que recoge los datos personales: edad, condición civil, semestre y carrera que cursa, lengua materna que habla o segunda lengua, lugar de origen, modalidad y nivel cursado de sus estudios, y cuáles han sido las formas de cómo aprendieron a escribir el español, así como las debilidades u obstáculos que presentaron para elaborar un texto escrito en su área de especialización.

En la segunda parte del instrumento se les pidió que escribieran de manera espontánea una historia, un suceso o una experiencia emotiva con la que se evidencie el manejo de manera progresiva de cuestiones gramaticales, morfosintácticas, semánticas y ortográficas, que son reglas lingüísticas que permiten dar cuenta del nivel de dominio que pueden tener en el manejo de la competencia comunicativa y lingüística con el enfoque intercultural (Casanny y Sanz, 2000; Gómez, 1997).

Para el análisis y comprensión de la información que se extrajo del instrumento que se aplicó a 83 estudiantes de las dos instituciones, los datos se procesaron para identificar los rasgos de los textos en su base morfológica, sintáctica, ortográfica y semántica, tomando en cuenta el modelo gramatical de artículo, sujeto, verbo y predicado. 
En lo sintáctico de las oraciones, se valoró el respeto de las reglas adecuadas para dar estructura a un texto, utilizando el uso de sujeto, verbo, predicado, singular, plural, conectores, entre otros para dar claridad y coherencia a los textos escritos.

Con los datos recabados se intentó relacionar el nivel de dominio de la comunicación escrita que estos grupos de jóvenes poseen para conocer si se corresponde con lo que se propone en los perfiles de egreso de ambos programas educativos.

En esta actividad, se pidió al estudiantado que plasmara con la mayor honestidad las respuestas a los dos apartados, se otorgó una hora y media para que contestaran el instrumento. El cuestionario fue aplicado en los horarios matutino y vespertino, además de los fines de semana que manejan ambas instituciones educativas; se brindó el formato de consentimiento informado y la totalidad de estudiantes estuvo de acuerdo en participar en la investigación. Decidieron escribirlo en español y, aunque estaban en libertad de escribirlo en la lengua de origen, nadie lo hizo.

\section{Análisis y discusión de resultados}

\section{Las características escolares y étnicas del estudiantado}

A partir de los datos analizados de 83 cuestionarios que fueron aplicados a estudiantes que se forman con el enfoque intercultural, se encontró que $75.9 \%$ es de origen indígena y $24.10 \%$ mestizo; 51 pertenecen a la región Altos Tsotsil-Tseltal, 8 a la región de los Bosques, 8 a la región Metropolitana, 7 a la región Mezcalapa, 6 a la región de Los Llanos, 2 a la región de la Meseta Comiteca Tojolabal, y 1 a la región Selva Lacandona ${ }^{8}$, como se muestra en la figura 1.

De los grupos estudiantiles encuestados, $54.21 \%$ fueron hombres y $45.78 \%$ mujeres: 40 estudiantes cursan la Licenciatura en Lengua y Cultura en el cuarto y sexto semestre de la UNICH; 43 de la UPN (21 hombres y 22 mujeres) que cursan la licenciatura en Educación Preescolar y Primaria Indígena en sexto y séptimo semestre inscritos en el Plan LEPEPMI 1990.

Del total de participantes, 23 son hablantes de la lengua tsotsil, 28 son del tseltal, 20 español, 8 zoques, 2 ch'ol y 2 del grupo étnico mam. En ese sentido, 63 tienen como lengua materna alguna de las lenguas mayas o zoque, mientras que 20 tienen como lengua materna el español. En el caso de estudiantes que tienen como lengua materna el español están en vías de aprender alguna de las lenguas mayas que se hablan en las regiones de Chiapas, como parte de su proceso de formación profesional, como se muestra en la figura 2.

El $74.7 \%$ de estudiantes dijo ser soltero, mientras que el $25.3 \%$ es casado por la religión católica o no católica.

8 La ubicación de residencia de estudiantes de manera formal y que aparecen adscritos a cada región económica se hizo a partir del reordenamiento territorial elaborado por el Gobierno del Estado de Chiapas a través de la Secretaria de Hacienda y Crédito Público para fines de la división política territorial. El análisis que se hizo de los lugares de adscripción de los estudiantes se basó en esta información contenida en la página web que fue consultada el día 25 de abril de 2013. Para mayor información véase página el documento de Clasificación municipal y regional http://www.haciendachiapas.gob.mx/marco-juridico/Estatal/informacion/Lineamientos/ Normativos/2013/XII-Clas-Mpal-Regional.pdf 


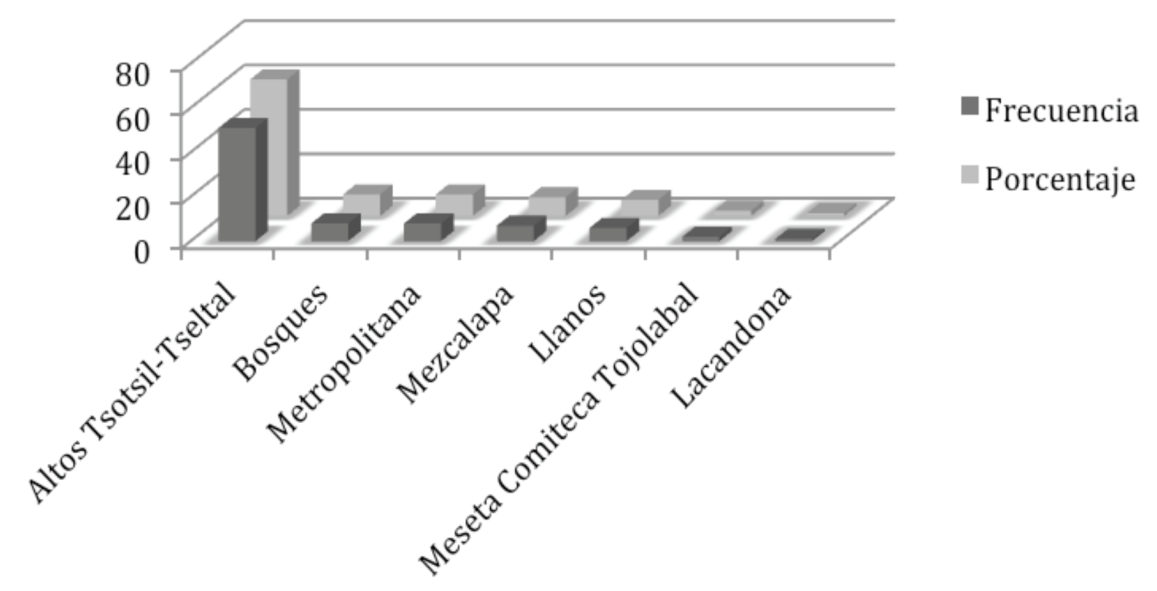

Figura 1: Regiones de procedencia de estudiantes. Cuestionario de datos generales.

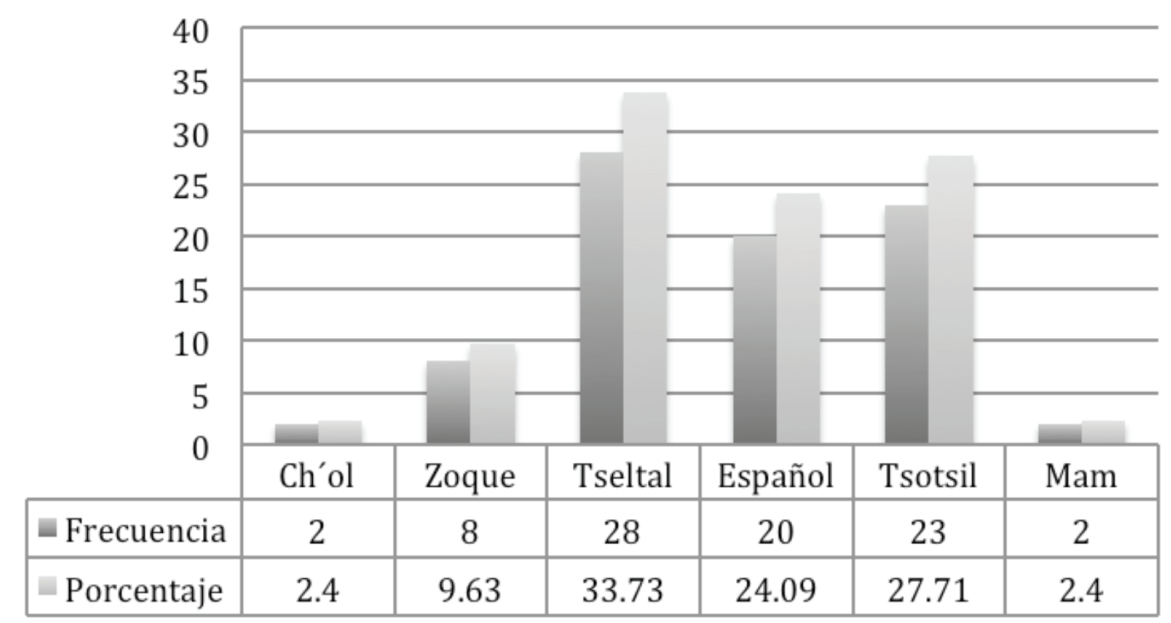

Figura 2. Lengua materna de participantes. Cuestionario de datos generales.

El 54.22\% de los 83 estudiantes cursó sus estudios de primaria en el subsistema de educación general en el idioma español, mientras que $45.78 \%$ de jóvenes cursó sus estudios en una escuela primaria del subsistema de educación indígena, donde el currículo contempla la enseñanza de lectura y escritura en la lengua materna.

El 83.1\% de alumnos y alumnas señaló que optó por estudiar en la modalidad de secundaria general, $10.84 \%$ en el subsistema de educación secundaria técnica, $6.06 \%$ en telesecundaria, por lo que en ninguna de estas modalidades educativas tuvieron la enseñanza de la escritura de la lengua materna. Con respecto a la educación media superior, 71.24\% cursó sus estudios en alguna de las preparatorias del Estado, 15.66\% en los colegios de bachilleres 
de Chiapas (COBACH), 2.4\% en los colegios de estudios científicos y tecnológicos de Chiapas (CECyTECH), 7.2\% en el Centro de Bachillerato Tecnológico y Agropecuario (CBTA), y 3.6\% de estudiantes en la modalidad de telebachillerato. En ninguna de estas modalidades las actividades de enseñanza incluyeron el uso de la lengua materna indígena (tabla 1).

Tabla 1

Trayectoria de formación en español y lengua indigena

\begin{tabular}{lrr}
\hline & $\begin{array}{l}\text { Enseñanza en } \\
\text { español }\end{array}$ & \multicolumn{2}{l}{$\begin{array}{l}\text { Enseñanza } \\
\text { bilingüe }\end{array}$} \\
\hline Primaria & $54.22 \%$ & $45.78 \%$ \\
\hline Secundaria & $100 \%$ & 0 \\
\hline Preparatoria & $100 \%$ & 0 \\
\hline
\end{tabular}

Este dato resulta de sumo interés, dado que permite entender lo que ha sucedido, por una parte con el trabajo de lograr el dominio hablado y escrito del español; pero también lo que tiene que ver con la recuperación de los elementos propios de su cultura a partir de la preservación de la lengua materna.

En cuanto a la edad, el $39.7 \%$ de estudiantes de la UNICH se encuentra en el rango de 19 a 23 años, en tanto que $7.2 \%$ tiene entre 24 a 26 años. Mientras que en la UPN la edad varía considerablemente, ya que $34.9 \%$ de estudiantes tiene entre 22 y 30 años y $18.2 \%$ entre 31 y 52 años.

\section{La estructura gramatical, sintáctica, morfológica y semántica de los textos escritos}

De 83 alumnos, hombres y mujeres, que participaron elaborando un texto escrito, encontramos que $72.2 \%$ de los trabajos presenta una descripción legible de sus ideas, mientras que $27.7 \%$ de ellos no las logra reflejar con claridad, al momento de escribir los enunciados de sus escritos.

De los trabajos que aparecen con una escritura legible, solo 20 presentan una motricidad delgada y centrada, ${ }^{9}$ que hace que los textos posean un buen nivel de aceptación estética y de lectura. Adicionalmente a la motricidad delgada que presentan los escritos de este grupo de jóvenes, se encontró que las letras y las palabras no gozan de una buena direccionalidad, unas

9 Por motricidad delgada se refiere a la condición psicológica de que tienen un escrito uniforme y fino al momento de plasmar las palabras en la hoja de libreta. Ubica con claridad las letras en las hojas del cuaderno. 
son garabatos y otras al tiempo de plasmarlas no logran mantener el equilibrio en la escritura, por eso las letras aparecen torcidas al momento de verlas en la hoja del cuaderno o de la libreta.

En la elaboración de un párrafo se detecta que un 90.36\% (f. 75) de estudiantes no concluye sus ideas con claridad en el trabajo, se encuentra con dificultades para conectar las ideas o determinar de manera organizada las frases que aluden a una oración indeterminada en la construcción del párrafo o de todo el texto elaborado, en tanto que un 9.63\% (f. 8) logra este proceso de conclusión de sus ideas. Como consecuencia, se dificulta, para cualquier persona que lo lea, comprender el desarrollo integral del tema.

Con respecto al manejo de los signos de puntuación existe un limitado y errático uso de estos (punto y seguido, punto y aparte, comas, dos puntos en las oraciones cortas), se observa una redacción en párrafos muy largos, que involucran varias ideas que no logran conectarse adecuadamente, lo que provoca que al leer se limite la comprensión del texto. Esta situación se presentó en un 87.95\% (f. 73) de estudiantes, dando lugar a la confusión en la idea central y las ideas secundarias que acompañan y dan sentido a la primera.

Existe una ausencia de los usos de las reglas ortográficas para la producción de un texto. En el $90.36 \%$ (f. 75) de los textos elaborados por el estudiantado se observa una confusión en el uso de la c con s, la v o la b, el uso de la h, de los signos de interrogación o exclamación, cometiendo errores en palabras como a cerca en lugar de acerca, con migo en lugar de conmigo, acabo en lugar de a cabo, indicando con esto la deficiencia en el empleo de las reglas ortográficas.

Con respecto al nivel semántico, los textos no reflejan una descripción que aluda a explicaciones de mayor madurez cognitiva; por el contrario, se enuncian diversas oraciones descontextualizadas, que no logran plasmar en el texto con un vocabulario pobre, al no utilizar sinónimos y repetir constantemente las mismas palabras en un solo párrafo. En las diversas oraciones que emplean, se observa que utilizan un vocabulario común de uso cotidiano; sin embargo, mezclan algunas palabras con términos técnicos, lo que produce una pérdida de textura, calidad y fluidez de los enunciados en la construcción de los textos.

\section{Títulos y temas de un texto escrito desarrollados por los alumnos y alumnas}

Ante la posibilidad de realizar un escrito de forma espontánea, el 55.42\% de estudiantes concluyó la tarea solicitada de construcción completa del texto, el resto no lo hizo y argumentó que no tenían la imaginación ni los elementos para poder llevarlo a cabo. Aun sin ser concluidos, todos los textos fueron sometidos a revisión. De los textos concluidos, establecieron los temas y dieron nombre a los artículos en el escrito libre que se les solicitó, encontrando que hubo mayor coincidencia en los de moraleja, tradiciones y costumbres, así como los personales, como se observa en la Tabla 2. 


\section{Tabla 2}

Tema y título de los escritos libres de los estudiantes

Aventura

1. El hombre que encontró un tesoro en el camino

2. La historia de dos niños

total 2

Amor

1. La historia de una relación amorosa

2. La bella y la bestia

3. La vida y la novia

total 3

Tradiciones y costumbres

1. La cultura indígena tseltal

2. La explotación entre los indígenas

3. El bosque. Las historias en la comunidad

4. El volcán Chichonal

5. El sombreruro

6. Las zonas arqueológicas

7. Bochil mi pueblo, mi orgullo de ser indígena

8. El campesino

total 8

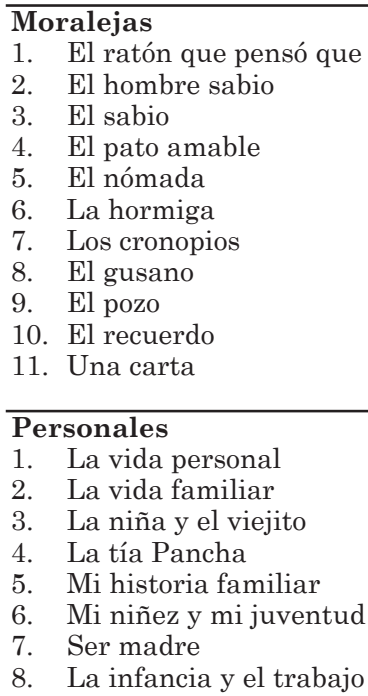

total 11

Escolares

1. Una historia escolar

2. La historia de ingreso al magisterio

3. Cómo ser una instructora comunitaria

4. Cómo ser una maestra bilingüe

5. La carrera de maestra indígena

6. La escuela Emiliano Zapata

total 6

Problemáticas sociales

1. La globalización

2. La migración de campo a ciudad

3. El movimiento de 1994

4. La discriminación

5. Los partidos políticos y la división comunitaria

Motivacionales

total 5

1. La sobrevivencia

2. El sufrimiento

3. La adversidad y la lucha

total 3

Nota: Elaboración propia (2014). 
El alumnado retrata, a través de sus escritos, las implicaciones personales y sociales que ha tenido que pasar para poder llegar a la escuela y permanecer en ella. Percepciones de cómo debe ser la vida en la familia, donde imprimen valores como la honestidad, la buena convivencia, ser una buena persona responsable y puntual.

Otro grupo de escritos hace referencia a las tradiciones de las fiestas patronales, la organización de la vida en la comunidad, los valores y conocimientos sobre organización social, experiencias locales, regionales y nacionales, de cómo se puede vivir en la comunidad y de la relación que guarda el medio ambiente, el cuidado de los recursos forestales y su hábitat.

Se encontraron también temas cuyo contenido aborda la presencia de los partidos políticos y sus implicaciones ideológicas en la lucha por el poder e intercomunitarias que derivan en conflictos y violencia; las enfermedades y el clima de violencia tanto en la casa como en la vida cotidiana, así como las subjetividades místicas y mágicas en el control social y cultural que ejercen las deidades en la comunidades indígenas y no indígenas. Además, las descripciones señalan diversas estrategias de sobrevivencia que hacen las familias de las comunidades indígenas y no indígenas.

En los textos escritos sobresalen las historias personales, donde se derivan sus experiencias difíciles y dolorosas enfrentadas para estudiar la primaria, secundaria y preparatoria hasta llegar a la universidad. Las implicaciones socioculturales de salidas de las familias de la comunidad de origen a la ciudad; el racismo latente en las escuelas por no hablar el español y por hablar la lengua indígena; los esfuerzos que están haciendo su padres para que estudien una carrera; las experiencias escolares de cómo están cursando una carrera para llegar a tener una estabilidad laboral y conseguir un ingreso económico remunerable en tiempos de crisis laboral; las decepciones amorosas que han experimentado en el transcurso de sus vidas, el sentido de la responsabilidad en el trabajo, en la escuela y la familia.

Otro grupo menor de textos alude a historias de ciencia ficción en donde se visualizan estilos de vida del mundo moderno, recreando figuras artificiales de animales que pueden hablar y que conjugan experiencias de realidades ficticias.

\section{Apreciaciones sobre la enseñanza de la escritura en español}

La enseñanza del español en contextos educativos en donde la mayoría del estudiantado tiene como lengua materna una propia de su comunidad indígena presenta muchas complejidades. En el caso del estudiantado participante en este estudio, a través de entrevistas breves, la mayoría coincidía en señalar que su acercamiento a la escritura en español se dio mediante la aplicación mecánica de ejercicios, es decir, se llevó a cabo a través de repeticiones escritas (planas) implementadas por sus docentes, estrategia que se fundamenta en la memorización y la asociación de imágenes y figuras; sin embargo, esta habilidad es limitada para explorar la compresión que el alumnado tiene sobre un contenido.

Una parte de estudiantes mencionó que las reglas de la escritura que aprendieron en la escuela durante los primeros años consistieron en el uso de los acentos y las comas. Recuerdan que el aprendizaje de las reglas ortográficas y fonológicas formaba parte de los contenidos de la clase de español (palabras agudas, graves, esdrújulas y sobreesdrújulas), la enseñanza buscaba 
que cada estudiante pudiera conocer los procedimientos fonológicos para la acentuación de las oraciones de español. Quienes tenían una lengua materna indígena coinciden en señalar que les fue muy difícil aprender en el aula de manera ordenada las reglas ortográficas y gramaticales, que a lo largo de toda su trayectoria escolar fueron temas recurrentes.

Para la mayoría del estudiantado elaborar las planas ${ }^{10}$ como tarea cotidiana de aprendizaje lectoescritor, tenía como propósito que pudieran escribir a partir del conocimiento de las vocales y del abecedario. En estos ejercicios se hacía énfasis en su pronunciación, lo que resultaba complicado cuando pasaban a leer en voz alta frente a sus compañeros en el aula. Los grupos de estudiantes indígenas en su mayoría admitieron que fue un proceso muy doloroso aprender a escribir y a leer el español. Además, manifiestan que no ha sido un proceso escolar completo, porque aún continúan con serias carencias cognoscitivas para redactar una carta o un mensaje en español. Reconocen que las actividades de enseñanza se centraban en un ejercicio mecánico sin interacciones o explicaciones, donde sus docentes generalmente les exigían "escribir, leer y hablar bien el español", así como hacer transcripciones de planas de textos que se encontraban en los libros de español.

En cuanto al alumnado que tiene como lengua materna el español, coincide en que en la escuela primaria se les enseñaba también con planas que no tenían sentido educativo porque debían copiar textualmente las historias. Lo anterior trajo como resultado que no aprendieran a redactar respetando la gramática y la ortografía, por ejemplo, desconocen dónde llevan acento las palabras.

En la educación primaria y secundaria la apropiación de las competencias para el dominio escrito y oral del español se traducía en la ejecución de ejercicios de manera mecánica. Una estrategia que buscaba mejorar la grafía de los estudiantes era el dictado, que consistía en la realización de hasta cinco planas diarias con el fin de que valoraran las letras y memorizaran las reglas de escritura. En estas actividades era frecuente que cuando no realizaban correctamente los ejercicios (letras ilegibles, no respetar los espacios entre letras, borrones y manchados) sus profesores o profesoras les regañaban o castigaban físicamente.

Con respecto a la formación en el nivel medio superior, los grupos de estudiantes indígenas relatan, de forma oral, que se leía mucho en español, pero que debido a su deletreo por una pobre fluidez lectora no lograban comprender la totalidad del contenido de los textos leídos grupalmente en el aula, situación no prevista ni intervenida por el profesorado, sino como una variable que impedía el aprendizaje.

Mencionaron que en sus trayectorias escolares no recordaban cómo era la ejecución de ninguna estrategia didáctica de escritura en español que les resultara atrayente. También consideraron que no hay fórmulas y mucho menos recetas que les hayan permitido adquirir de manera sólida la competencia escrita del español. Se encontró que en la educación primaria los cuerpos docentes ordenaban a sus estudiantes iniciar las actividades localizando un tema que pudiese ser desarrollado, posteriormente que colocaran un título y terminaran desarrollando el contenido del texto. En algunas ocasiones recurrían a materiales didácticos para que los alumnos y las alumnas observaran cómo se elaboraba un signo gráfico; pero, al mismo tiempo, admitieron que fue un proceso muy complicado aprender de manera sistemática la adecuada utilización de los signos de puntuación y las reglas ortográficas.

10 La tarea de planas consiste en copiar literalmente lo mismo, cuantas veces indicara el profesor o profesora hasta completar las hojas o los cuadernos. Este sistema es arbitrario y sin sentido por el mero acto de la repetición. 
Para lograr un buen trazo gráfico, recuerdan que sus docentes de primaria, les tomaban de la mano y posteriormente les pedían que lo reprodujeran por cuenta propia en el proceso de iniciación de la escritura. Cuando había dominio de la grafía y su significado, les pedían que escribieran cuentos e historias de la localidad para después ser sometidos a la corrección ortográfica. A veces las clases de español eran muy aburridas, porque no había una forma de estimular el aprendizaje para el desarrollo de la competencia lectoescritora en el estudiantado.

Señalaron que por lo general su estancia en la secundaria y el bachillerato, en sus diversas modalidades y niveles ${ }^{11}$, no fue exitosa, ya que la enseñanza recibida resultó ineficiente, tanto para aprender la escritura del español como de la lengua indígena. Ocho estudiantes expresaron dar gracias al profesorado porque aprendieron a escribir con reglas ortográficas y signos de puntuación. Agregaron también que de acuerdo con la enseñanza que cursaron ahora pueden leer, escribir y continuar estudiando en la universidad; pero esta apreciación la comparte una pequeña minoría del total de la población que participó en este estudio.

Los grupos de estudiantes indígenas admitieron tener buenos maestros y maestras a lo largo de la enseñanza en español, a pesar de los regaños y llamadas de atención que recibieron mejoraron su aprendizaje de la lengua escrita y oral. Por otra parte, los mestizos señalaron que, si bien es cierto en las clases predominaban los dictados, planas y muchos ejercicios memorísticos de escritura, desde el primer grado ya sabían leer, escribir y hacer las operaciones aritméticas.

El 90\% de estudiantes admitió no tener gusto por la lectura, reconocen que han llegado a la universidad sin el estímulo afectivo con el que apreciar y valorar la lectoescritura como parte del desarrollo profesional para las exigencias del mundo laboral.

Se logró identificar que, del total, diez usan de manera sencilla estrategias de comprensión lectora, tales como la anticipación, el resumen y la síntesis, que aprendieron al combinar adecuadamente el uso del español con su lengua materna.

Los textos escritos producidos por la mayoría de estudiantes evidencian la recuperación de aprendizajes previos escolares con deficiencias. En su mayoría, no poseen un dominio total de los signos ortográficos y de acentuación, aunque se encuentren en la universidad aún no han podido superar estas deficiencias. El 75\% de los escritos redactados denota no haber alcanzado a comprender el sentido de la pregunta en sus respuestas, puesto que la mayor parte admite ser conscientes de las problemáticas que involucra el ser pobres lectores o lectoras, aunque no vislumbran posibles estrategias que debieran llevar a cabo con lo que denominan como debilidades, aunque están conscientes de que el dominio de la lecto-escritura académica es una exigencia del nivel en donde se están formando

\section{Discusión}

Los trabajos inherentes a la elaboración de textos y a los hallazgos encontrados en la población estudiantil de la UNICH y la UPN dan cuenta de que el estudiantado no ha podido apropiarse de las competencias escritas y orales que le permitan incrementar la calidad de los aprendizajes y no se ha beneficiado de la enseñanza con un modelo intercultural.

11 Telesecundaria, secundaria técnica, secundaria general, Colegio de Bachilleres de Chiapas, Centro Estudios Tecnológicos y Agropecuarios, telebachillerato, Centro de Estudios Científicos y Tecnológicos de Chiapas, Preparatoria del Estado. 
El aprendizaje del uso de signos ortográficos y de puntuación, así como la ausencia de niveles de articulación de las ideas, están relacionados con ła pobre alfabetización que ha experimentado la juventud que proviene de poblaciones indígenas de la región Altos de Chiapas. Los grupos de estudiantes indígenas han adquirido un bilingüismo sustractivo que ha reforzado que el español tenga una fuerte presencia en los procesos de comunicación en detrimento de las prácticas sociales de comunicación propias. Resulta importante que las estrategias metacognitivas tanto personales como colectivas se realicen, ya que contribuyen a la adquisición de habilidades inferenciales asociadas al dominio de textos académicos y especializados, y facilita la comprensión a nivel micro, macro y superestructural del texto, necesario en el ámbito de la formación de las futuras generaciones profesionales (CisnerosEstupiñán, Olave-Arias y Rojas-García, 2012; Venegas, 2007).

El dominio de habilidades de escritura y de comprensión lectora en los tiempos modernos es un imperativo social que la educación superior impulsa; sin embargo, su incidencia en la formación de estudiantes atraviesa omisiones en la formación profesional y se requiere del desarrollo de la competencia comunicativa bilingüe equilibrada, necesario para estimular el desarrollo de las lenguas originarias y del español, para que el alumnado mejore su desempeño académico en la Universidad Intercultural y la Universidad Pedagógica. Este esfuerzo implica impulsar metodologías didácticas participativas entre estudiantes y docentes, con el fin de situar a la universidad como el lugar fundamental para la conquista del poder de participación en las tradiciones escritas de las ciencias, las filosofías y las literaturas desde sus diversos orígenes culturales (Castedo y Zuazo, 2011).

La formación de maestros y maestras para la enseñanza del español y de la lengua indígena (enseñanza bilingüe) se ha constituido en uno de los obstáculos más importantes para el desarrollo de una verdadera educación intercultural. Estudios sobre este tipo de aprendizaje en regiones étnicas en relaciones de comunicación diglósica sugieren que se elaboren estrategias didácticas que ponderen el uso de la lengua en su forma escrita u oral, también que se recuperen las tradiciones cognitivas de la cosmovisión y la valoración de la identidad étnica para que estos códigos de aprendizaje, con eficacia, permitan que las poblaciones estudiantiles se apropien de la lengua castellana y mejoren el nivel de eficiencia lingüística en ambas lenguas (Hamel et al., 2004).

Los grupos de estudiantes que participaron en este estudio señalan que las clases que se imparten cotidianamente son monótonas y, si se le agrega la apatía sobre la lectura, los avances son mínimos. Consideramos que el limitado logro en el diseño de pedagogías interculturales apela a una realidad educativa en donde la formación profesional de alumnos y alumnas está permeada por conflictos interculturales (jerarquías lingüísticas entre culturas) que requieren ser atendidos para mejorar las competencias escritas y orales.

Las propuestas para la formación intercultural proponen que las lenguas sean el punto de intersección para el diálogo intercultural; sin embargo, la forma en la que se desarrolla la enseñanza escrita del español no coadyuva a este fin, ya que el estudiantado se encuentra ante el imperativo de desempeñarse en un mundo laboral en donde se requiere de un dominio eficiente del español. No obstante, al no contar con estas competencias plenamente desarrolladas, experimenta la discriminación del sistema educativo ya que se incorpora al campo laboral sin 
las experiencias necesarias para un desempeño competitivo frente a otros grupos profesionales de su mismo campo. La escritura es el capital cultural de mayor importancia social y educativa, necesario para la construcción de relaciones de respeto y tolerancia en las universidades; no obstante, en este modelo intercultural, las lenguas y su enseñanza no tienen la misma jerarquía, por lo tanto, su dominio es desequilibrado (Concha, Aravena, Coloma y Romero, 2010; Cueva, 2008; Duque, Vera y Hernández, 2010). No existe una política bilingüe.

Con estos hallazgos, todo parece suponer que los objetivos del perfil de egreso distan mucho de las prácticas concretas de comunicación escolar de alumnos y alumnas al producir textos descriptivos que les permitan acceder al mundo de la comunicación y de la sociedad del conocimiento. Las implicaciones del desarrollo del currículo indican que los trabajos previos que ha logrado construir el estudiantado denotan carencias de orden estructural que se relacionan en los ámbitos gramatical, lexical, semántico y sintáctico, necesarios para la construcción de procesos de producción de textos escritos y de la comprensión lectora (Figari, 2010 y García, 2010).

Esta disyuntiva escolar que se presenta en el área del lenguaje para la apropiación de una alfabetización está relacionada, posiblemente, con el hecho de que los grupos de estudiantes nunca tuvieron la oportunidad de pasar por un proceso de alfabetización en lengua materna; sino, por el contrario, el español se les impuso como segunda lengua desde los procesos de escolarización, razón por la cual los problemas de orden de estructuración de un texto siguen siendo consecuencia de las reminiscencias del pasado acerca del proceso de castellanización que experimentaron los pueblos indígenas en el marco de la educación nacionalista (Aveledo y Martins, 2009; Bastiani, Ruiz-Montoya, Estradada, Cruz, Aparicio y Bermúdez, 2013).

Entre los grupos de las generaciones futuras de docentes indígenas en servicio se presentan dificultades en la elaboración de textos descriptivos y en el manejo adecuado de normas gramaticales, morfosintácticas y ortográficas, vitales en la conformación de competencias comunicativas para poder actuar en la sociedad del conocimiento. En el perfil de egreso de la LEPEPMI'90 se valora que las prácticas escolares puedan interactuar de manera comunicativa bilingüe en la lengua indígena y el castellano para mejorar sus habilidades comunicativas. Sin embargo, los talleres cocurriculares en las trayectorias formativas pueden ser un mecanismo de apoyo para la alfabetización bilingüe universitaria.

Ambas instituciones tienen un reto mayúsculo: la formación intercultural en el área de lenguaje, toda vez que las prácticas escolares evidencian problemas sociolingüísticos y psicolingüísticos que obstruyen el desarrollo de la educación indígena intercultural.

La semipresencialidad de estudiantes de la UPN (cada quince días) pudiera ser un factor adicional que complejiza la situación que viven en el desarrollo de las competencias comunicativas; sin embargo, esto también sucede con los grupos de la UNICH que acuden diariamente a clases, por lo que habría que pensar que, en el fondo, el verdadero problema se centra en una enseñanza deficiente, porque no se han recuperado sus experiencias previas bilingües para fortalecerles en el nivel superior. Es evidente que la formación hasta ahora recibida no ha colocado a estos grupos como eficientes interlocutores del uso social de un lenguaje escrito y verbal en el que puedan competir comunicativamente en el mundo de la sociedad nacional. 


\section{Conclusiones}

En el campo de la educación superior resulta necesario -tanto para el estudiantado de la Universidad Intercultural de Chiapas en San Cristóbal de Las Casas, como para el de la Universidad Pedagógica Nacional, Unidad 071, de Tuxtla Gutiérrez, Chiapas, que se redefinan los procesos de formación para que el español y la lengua indígena se constituyan en eje transversal para asegurar que las trayectorias formativas de los alumnos y las alumnas adquieran las habilidades de comunicación escrita.

Los resultados del trabajo de investigación denotan que la política educativa de formación docente intercultural en ambas instituciones educativas debe apuntalar un proceso formativo comunicativo que dé cuenta de un aprendizaje de estrategias metacognitivas para superar las debilidades de carácter morfológico, sintáctico, fonológico y de acentuación que evidencian trayectorias escolares que no le ha permitido establecer, a su alumnado, un verdadero proceso de apropiación de la escritura en la elaboración de textos escritos. La escritura del español y de la lengua indígena es una necesidad en el mundo contemporáneo, por lo que personal docente e instituciones de educación superior deben reencauzar la formación de estudiantes indígenas que no han recibido una alfabetización -ni en lengua indígena ni en español- de manera sistemática.

El modelo intercultural requiere de una política de educación superior en esta materia, que enfatice la enseñanza del español y de la lengua indígena para que sus estudiantes puedan entablar el diálogo respetuoso entre culturas en un mundo globalizado.

En ambas instituciones de educación superior, el papel educativo que debe accionar en ámbitos de formación escolar de jóvenes indígenas debe crear las condiciones organizativas, pedagógicas y financieras para promover una cultura de la actualización docente que permita la apropiación de elementos metodológicos para encauzar una formación escolar de forma congruente y pertinente en los ámbitos educativos interculturales. En este proceso de habilitación docente, el trabajo didáctico en el aula procurará que los alumnos y las alumnas adquieran las competencias con las que puedan, desde sus realidades étnicas, escribir y comunicar la cultura indígena para promover el desarrollo de las entidades étnicas originarias como parte de la política educativa de educación superior intercultural.

El dominio de destrezas de escritura implica que la política educativa se traduzca en el ámbito práctico de las vidas concretas de las aulas universitarias en saberes de orden gramatical, morfológico, sintáctico y semántico; en donde los grupos de estudiantes de ambas universidades contribuyan al desarrollo de expectativas y actitudes interculturales para que, desde la cultura de la comunicación escrita, se traduzcan los esfuerzos institucionales en un proceso de calidad educativa que exige el mundo contemporáneo globalizado.

Las acciones que se realicen en las universidades deberán estar dando respuesta a una preocupación de las comunidades originarias sobre las generaciones de estudiantes que aún no han podido escolarizarse, para que puedan concluir, en el marco de las relaciones interculturales, un proceso de formación integral en educación superior intercultural superior.

Es necesario que se establezca un programa de formación de actualización docente intercultural, en donde personal de asesoría académica de ambas licenciaturas conviertan 
el análisis de la práctica docente en una condición necesaria que contribuye a elevar los niveles de habilitación pedagógica para asegurar la formación de estudiantes en el campo de la comunicación escrita y se posibilite, así, la adquisición de un bilingüismo equilibrado favorecedor de ambas lenguas y de la cultura local, frente a las demandas sociales y educativas de los escenarios escolares contemporáneos a nivel local, regional, nacional e internacional.

\section{Referencias}

Abajo Alcalde, J. E. y Carrasco Pons, S. (2011). La situación escolar del alumnado de minorías étnicas: El modelo explicativo ecológico-cultural de John Ogbu. Recerca, 11, 71-92. Recuperado de http://www.e-revistes.uji.es/index.php/recerca/article/view/227/209

Areizaga Orube, E. (2000). El enfoque comunicativo. Propuestas didácticas. En U. Ruiz Bikandi (Ed.), Didácticas de segundas lenguas en educación infantil y primaria. Madrid: Síntesis educación.

Aubry, A. (2011). Otro modo de hacer ciencia. Miseria y rebeldía de las ciencias sociales. En B. Baronnet, M. Mora Bayo y R. Stanhler-Sholk (Coords.), Luchas "muy otras". Zapatismo $y$ autonomía en las comunidades indigenas de Chiapas (pp. 59-78). México, D.F.: Universidad Autónoma Metropolitana, Xochimilco (UAM), Centro de Investigaciones Sobre Antropología Social (CIESAS), Universidad Autónoma de Chiapas (UNACH). Recuperado de https://zapatismoyautonomia.files.wordpress.com/2013/12/luchas-muy-otras-2011.pdf

Aveledo, F. y Martins, I. (enero-junio, 2009). La adquisición de las clausulas interrogativas parciales en el español infantil: ¿Reglas innatas o fórmulas aprendidas? Boletín de lingüistica, 21(31), 5-35. Recuperado de http://www.redalyc.org/articulo. oa? id $=34711680001$

Bastiani, J., Ruiz-Montoya, L., Estrada, E., Cruz, T., Aparicio, J. Y Bermúdez, F. (2013). Medición de conocimientos con reactivos en ch"ol y español en niños de educación básica con modelos pedagógico intercultural bilingüe. Revista Electrónica de Investigación Educativa 15(1), 107-120. Recuperado de http://redie.uabc.mx/vol15no1/contenidobastianietal.html

Bertely-Busquets M. (2002). Conociendo nuestras escuelas. Un acercamiento etnográfico a la cultura escolar, México D.F.: Paidós.

Cassany, D., Luna, M. y Sanz, G. (2000). Enseñar lengua. Barcelona: Editorial Grao. Recuperado de http://beceneslp.edu.mx/PLANES2012/50\%20Sem/04\%20Producci\%F3n\%20de\%20 textos\%20escritos\%20\%28prim\%29/Materiales/Unidad\%20I/02\%20Complementarios/ Ense\%F1ar\%20lengua\%20-\%20Daniel\%20Cassany\%20et\%20al.pdf 
Castedo, M. yZuazo, N. (2011). Culturas escritas y escuela: Viejas y nuevas diversidades. Revista Iberoamericana de Educación, 4(56), 1-14. Recuperado de http://www.rieoei.org/ deloslectores/4843Castedo.pdf

Cisneros-Estupiñán, M., Olave-Arias, G. y Rojas-García, I. (2012). Cómo mejorar la capacidad inferencial en estudiantes universitarios. Educación y Educadores, 15(1), 45-61. Recuperado de http://educacionyeducadores.unisabana.edu.co/index.php/eye/article/view/2130/2713

Concha, S., Aravena, S., Coloma, C. y Romero, V. (2010). Escritura expositiva en tres niveles de escolaridad: Coherencia y dominio de recursos lingüísticos. Literatura y lingüistica 21, 75-92. doi: http://dx.doi.org/10.4067/S0716-58112010000100007

Cueva, L. (noviembre, 2008). Estado de la cuestión sobre las características gramaticales de los marcadores discursivos del español. Forma y función 21, 87-106. Recuperado de http://www.redalyc.org/articulo.oa?id=21911525005

Duque-Aristizábal, C., Vera-Márquez, A. y Hernández-Gutiérrez, A. (2010). Compresión inferencial de textos narrativos en primeros lectores: Una revisión de la literatura. Ocnos: Revista de los estudios sobre la lectura 6, 35-44. Recuperado de https://www. revista.uclm.es/index.php/ocnos/article/view/190

Echeverría-Acosta, L. y McNulty-Ferri, M. (2010). Reading Strategies to Develop Higher Thinking Skills for Reading Comprehesion Profile Issues in Teachers Professionals Development [Estrategias de lectura para desarrollar habilidades de pensamiento más altas para el perfil de comprensión de lectura en profesores profesionales de desarrollo]. Profile, 12(1), 107-23. Recuperado de http://dialnet.unirioja.es/servlet/articulo?codigo=4858465

Fernández, J. (2005). Matriz de competencias del docente de educación básica. Revista Iberoamericana de Educación, 36(2), 1-14. Recuperado de http://www.rieoei.org/ investigacion/939Fernandez.PDF

Figari-Benítez, R. y García-Escala, G. (2010). El razonamiento analógico verbal: Una habilidad cognitiva esencial de la producción escrita. Onomázein, 2(22) ,165-194. Recuperado de http://www.redalyc.org/articulo.oa?id=134516603007

Frade-Rubio, L. (2009). Desarrollo de competencias en educación: Desde preescolar hasta el bachillerato. México, D.F: Inteligencia educativa.

García-Negroni, M. y Hall, B. (julio-diciembre, 2010). Escritura universitaria, fragmentariedad y distorsiones enunciativas propuestas de prácticas de lectura y escritura focalizadas en 
la materialidad lingüístico-discursiva. Boletín de Lingüística, 22(34), 41-69. Recuperado de http://www.redalyc.org/articulo.oa?id=34721061003

Goetz J. y LeCompte, M. (1988). Etnografía y diseño cualitativo en investigación educativa. Madrid: Editorial Morata.

Gómez-Palacio, M. (1997). La producción de textos en la escuela. México, D.F.: Secretaría de Educación Pública, Biblioteca de Actualización del Maestro.

Hamel, R. E., Brumm , M., Carrillo-Avelar , A., Loncon , E., Nieto , R. y Silva-Castellón, E. (enero-marzo, 2004). ¿Qué hacemos con la castilla? La enseñanza del español como segunda lengua en un currículo intercultural bilingüe de educación indígena. Revista Mexicana de Investigación Educativa, 9(20), 83-107. Recuperado de https://www.comie. org. $\mathrm{mx} / \mathrm{v1} /$ revista/portal.php?idm=es\&sec=SC03\&sub=SBB\&criterio=ART00403

López, L. (2003). ¿Dónde estamos con la enseñanza del castellano como segunda lengua en América Latina? En I. Jung y L. E. López (Comps.), Abriendo la escuela. Lingüistica aplicada a la enseñanza de lenguas (pp. 39-71). Madrid: Editorial Morata.

Pérez, M., Bellaton, P. y Emilsson, E. (2012). La enseñanza de las lenguas en México. Hacia un enfoque pluricultural.educ@upn.mx, Revista Universitaria. Recuperado de http:/l educa.upn.mx/hecho-en-casa/num-10/92-la-ensenanza-de-lenguas-en-mexicohacia-unenfoque-plurilingüe

Ruiz-Bikandi, U. (2000). La adquisición de la segunda lengua. En Uri Ruiz Bikandi (ed.), Didácticas de segundas lenguas en educación infantil y primaria (pp. ). Madrid: Síntesis educación.

Secretaría de Educación Pública (SEP). (1993). Plan y programas de estudio 1993. Educación Básica Primaria. México, D.F.: Secretaría de Educación Pública.

Secretaria de Hacienda y Crédito Público-Gobierno del Estado de Chiapas. (2013). Clasificación municipal y regional 2013. Secretaria de Hacienda y Crédito Público- Gobierno del Estado de Chiapas, Recuperado de http://www.haciendachiapas.gob.mx/marco-juridico/ Estatal/informacion/Lineamientos/Normativos/2013/XII-Clas-Mpal-Regional.pdf

SEP. (2004). Licenciatura en Educación Primaria con Enfoque Intercultural Bilingüe. Fundamentos y Estructura Curricular. Plan de Estudios 1997. México, D.F.: Secretaría de Educación Pública, Subsecretaría de Educación Básica, Dirección General de Normatividad, Dirección General de Educación Indígena, y Normal; Coordinación General de Educación Intercultural Bilingüe. 
SEP. (2008). Lengua originaria. Parámetros curriculares. Educación Básica. Primaria Indigena. México, D.F.: Secretaría de Educación Pública, Subsecretaría de Educación Básica, Dirección General de Educación Indígena.

SEP. (2010). ACUERDO número 540 por el que se actualiza el diverso número 181 por el que se establecen el Plan y los Programas de Estudio para la Educación Primaria, Poder Ejecutivo, Secretaría de Educación Pública, Diario Oficial, 20 de agosto de 2010, México, D.F.

SEP. (2011). Plan de estudios 2011. Educación Básica. México, D.F.: Secretaría de Educación Pública.

SEP-CGEIB. (2006). Universidad Intercultural de Chiapas. Modelo educativo, Secretaría de Educación Pública-Coordinación General de Educación Intercultural Bilingüe, México, D.F.

UNICH. (2007). Plan y programas de estudio de la licenciatura en Lengua y Cultura, San Cristóbal de Las Casas, Chiapas. México: Universidad Intercultural de Chiapas.

UNICH. (2012b). Informe estadístico de la oficina de Área de Control Escolar y el Departamento de Planeación de la UNICH. San Cristóbal de Las Casas, Chiapas, México: Universidad Intercultural de Chiapas.

Universidad Intercultural de Chiapas, UNICH. (2012a). Informe de rectoría enero-diciembre 2012, Universidad Intercultural de Chiapas, Recuperado de http://www.unich.edu.mx/ informe-rector

Universidad Pedagógica Nacional UPN. (1990). Plan y Programas de Estudios de Educación Primaria y Preescolar para el Medio Indígena, Plan 1990. México, D.F.: Universidad Pedagógica Nacional.

Venegas, R. (2007). Clasificación de textos académicos en función de su contenido léxico-semántico. Revista Signos, 40(63), 239-272. doi: http://dx.doi.org/10.4067/S071809342007000100012

Woods, P. (1998). La escuela por dentro. La etnografía en la investigación educativa. España: Paidós. 